\title{
Solving Becker's assortative assignments and extensions
}

\author{
F. Javier Martínez-de-Albéniz ${ }^{\mathrm{a}}$, Carlos Rafels ${ }^{\mathrm{a}}$, Neus Ybern ${ }^{\mathrm{b}}$ \\ ${ }^{a}$ Dept. de Matemàtica Econòmica, Financera $i$ Actuarial and BEAT \\ Universitat de Barcelona \\ Av. Diagonal 690, 08034 Barcelona, Spain \\ ${ }^{b}$ Dept. de Matemàtiques \\ Escola Politècnica Superior d'Enginyeria \\ Universitat Politècnica de Catalunya \\ Av. Víctor Balaguer 1, 08800 Vilanova i la Geltrú, Barcelona, Spain
}

\begin{abstract}
We analyze assortative assignment games, introduced in Becker (1973) and Eriksson et al. (2000). We study the extreme core points and show an easy way to compute them. We find a natural solution for these games. It coincides with several well-known point solutions, the median stable utility solution (Schwarz and Yenmez, 2011) and the nucleolus (Schmeidler, 1969). We also analyze the behavior of the Shapley value. We finish with some extensions, where some hypotheses are relaxed.
\end{abstract}

Keywords: Assortative market, assignment game, core, nucleolus

\section{Introduction}

Several economic and other areas depend heavily on the allocation of indivisible resources, objects or persons. A simple but deeply analyzed problem is that of matching, by making valuable pairs, the elements of two finite

\footnotetext{
${ }^{1}$ Financial support from research grant ECO2017-86481-P (Ministerio de Economía y Competitividad) is gratefully acknowledged, and from the Generalitat de Catalunya, through grant 2017SGR778.
} 
sets. These are referred to hereafter as the agents' sets. An assignment matrix or array collects all these valuations.

There are at least two main issues to be addressed in any assignment problem. Firstly we have to search/look and analyze how agents sort themselves out, that is, who is matched with whom. Secondly we have to solve the assignment problem by proposing and studying fair allocations for the agents. Both aspects have been dealt with extensively. Roth and Sotomayor (1990) serves as an accurate well-written text at these issues and an up-todate survey is Núñez and Rafels (2015).

Concurrent in time with the seminal paper of Shapley and Shubik (1972), Becker (1973) shows up an original application of assignment problems to the marriage market in which households produce some output to share between man and woman. In some assignment problems he conveys the effect of mating of the likes.

To model the mating of the likes he introduces two independent conditions on the assignment matrix. First, the association of likes is optimal when traits are complements and second, each trait has a monotone effect on the output, higher values have a larger effect. Moreover he proposes and analyzes the core of the game as a decisive reference solution set for searching allocations to solve the assignment problem. In Becker's paper no explicit allocation is proposed.

In Chapter 10, Open questions and research directions, Roth and Sotomayor (1990) already expresses the aim of studying special classes of assignment problems “(..) of the kind explored by Becker (...)" (page 247). Sherstyuk (1999) and Eriksson et al. (2000) manage to get insight into these topics. Sherstyuk analyzes multilateral supermodular assignment games. She proves the non-emptiness of the core and finds some extreme core allo- 
cations à la Shapley and Shubik. She imposes not only supermodularity but also monotonicity and opens a question about the possibility of relaxing the monotonicity condition, which we tackle in the last section of this paper. Eriksson et al. (2000) formalize Becker's ideas and introduce what is known as Becker's assortative assignment problems. Eriksson et al.(2000) discovers how to compute the buyers-optimal and sellers-optimal core allocations in the square case. They point out that all core payoffs rank the agents of any one side in the same way.

Finally Schwarz and Yenmez (2011) shows the existence of a family of central stable utility imputations for any assignment game. No formula is given and they open the question to analyze these allocations as a ponderation of the extreme core allocations. In particular, they show that for monotone supermodular production functions the fair allocation (Thompson, 1981) coincides with their median stable utility solution (MSUS).

The central purpose of this paper is to propose a new point solution to solve any assortative, square or not, assignment problem. It outcrops after a reexamination of the core of an assortative assignment game inspired by the seminal Becker's paper. Once defined and established an easy closed formula in terms of the original entries of the assignment matrix, we devote our efforts to intertwine its main properties.

Firstly we show that this solution always coincide with the median stable utility solution introduced in Schwarz and Yenmez (2011). Secondly it always coincides with the nucleolus (Schmeidler, 1969) of the assortative assignment game. This is a pleasant result since the nucleolus must be computed by tedious algorithms. Thirdly, we analyze the core and obtain an easy method to compute all extreme core points, and its number, a power of two. 
To end the paper, we analyze which are the conditions for the Becker's solution to coincide with the Shapley value (Shapley, 1953) and also some extensions of the model to cover other assignment markets where our results can be applied.

\section{Preliminaries}

A bilateral assignment market $(\mathcal{F}, \mathcal{W}, A)$ is formed by two non-empty disjoint sets, the set of firms $\mathcal{F}=\left\{f_{1}, f_{2}, \ldots, f_{m}\right\}$ and the set of workers $\mathcal{W}=\left\{w_{1}, w_{2}, \ldots, w_{n}\right\}$ where $m$ could be different from $n$ and a non-negative matrix $A=\left(a_{i j}\right)_{\left(f_{i}, w_{j}\right) \in \mathcal{F} \times \mathcal{W}} \in \mathrm{M}_{m \times n}^{+}$where each entry $a_{i j}$ represents some measure of the joint productivity of firm $f_{i}$ and worker $w_{j}$ when they are matched together. Clearly this productivity depends on the agents and could be written as $a\left(f_{i}, w_{j}\right)$ or $a(i, j)$. When $m=n$ the assignment market is said to be square.

A matching $\mu$ from firms $\mathcal{F}$ to workers $\mathcal{W}$ is a bijection from $F_{1} \subseteq \mathcal{F}$ to $W_{1} \subseteq \mathcal{W}$, such that $\left|F_{1}\right|=\left|W_{1}\right|=\min \{|\mathcal{F}|,|\mathcal{W}|\} .^{2}$ The set of all matchings is denoted by $\mathcal{M}(\mathcal{F}, \mathcal{W})$. Similarly we use $\mathcal{M}(S, T)$ to represent all matchings from $S \subseteq \mathcal{F}$ to $T \subseteq \mathcal{W}$.

We say firm $f_{i}$ and worker $w_{j}$ are matched by $\mu$ if $w_{j}=\mu\left(f_{i}\right)$ or $f_{i}=$ $\mu^{-1}\left(w_{j}\right)$. In this case we also say that each one is the partner by $\mu$ of the other one. With some abuse of notation we also write $\left(f_{i}, w_{j}\right) \in \mu$. When dealing with indices we also use $j=\mu(i)$ or $i=\mu^{-1}(j)$, if no confusion arises. When an agent does not have any partner by $\mu$ we say agent is unmatched.

\footnotetext{
${ }^{2}|F|$ represents the cardinality of the set $F$.
} 
A matching $\mu \in \mathcal{M}(\mathcal{F}, \mathcal{W})$ is optimal for the assignment market $(\mathcal{F}, \mathcal{W}, A)$ if for any other matching $\mu^{\prime}$ we have

$$
\sum_{\left(f_{i}, w_{j}\right) \in \mu} a_{i j} \geq \sum_{\left(f_{i}, w_{j}\right) \in \mu^{\prime}} a_{i j}
$$

The set of all optimal matchings is denoted by $\mathcal{M}_{A}^{*}(\mathcal{F}, \mathcal{W})$.

Shapley and Shubik (1972) associates any assignment market with a cooperative game, ${ }^{3}$ the assignment game, where the set of players is $\mathcal{F} \cup \mathcal{W}$ and the characteristic function $v_{A}$ is defined for any $\varnothing \neq S \subseteq \mathcal{F}$ and $\varnothing \neq$ $T \subseteq \mathcal{W}$

$$
v_{A}(S \cup T)=\max _{\mu \in \mathcal{M}(S, T)}\left\{\sum_{\left(f_{i}, w_{j}\right) \in \mu} a_{i j}\right\},
$$

where $\mathcal{M}(S, T)$ is the set of matchings from $S$ to $T$. Moreover $v_{A}(S \cup T)=0$ if either $S=\varnothing$ or $T=\varnothing$.

Notice that any pair of firm and worker evaluates its worth by exactly the corresponding matrix entry, and any other coalition determines its worth by pairwise combinations its members can form.

The agents of an assignment market may divide among themselves their worth in any way they like. An imputation is a non-negative vector $(x, y) \in$ $\mathbb{R}_{+}^{m} \times \mathbb{R}_{+}^{n}$ such that $\sum_{i=1}^{m} x_{i}+\sum_{j=1}^{n} y_{j}=v_{A}(\mathcal{F} \cup \mathcal{W})$, where we interpret $x_{i}$ and $y_{j}$ as the payoffs associated to firm $f_{i}$ and worker $w_{j}$ respectively.

The core of the assignment game $C\left(v_{A}\right)$ is described for any fixed $\mu \in$

\footnotetext{
${ }^{3}$ In a cooperative game $(N, v)$, the set of players is given by $N=\{1, \ldots, n\}$ and $v$ is a function that assigns a real number $v(S)$ for any coalition $S \subseteq N$ with $v(\emptyset)=0$. Its core is defined as $C(v):=\left\{x \in \mathbb{R}^{n} \mid \sum_{i \in N} x_{i}=v(N)\right.$ and for all $\left.S \subseteq N, \sum_{i \in S} x_{i} \geq v(S)\right\}$. A game is named balanced if its core is nonempty.
} 
$\mathcal{M}_{A}^{*}(\mathcal{F}, \mathcal{W})$ as those imputations $(x, y) \in \mathbb{R}_{+}^{m} \times \mathbb{R}_{+}^{n}$ satisfying

$$
\begin{aligned}
& x_{i}+y_{j} \geq a_{i j} \text { for all }\left(f_{i}, w_{j}\right) \in \mathcal{F} \times \mathcal{W}, \\
& x_{i}+y_{j}=a_{i j} \text { for all }\left(f_{i}, w_{j}\right) \in \mu .
\end{aligned}
$$

Unassigned agents by $\mu$ receive a zero payoff in any core allocation.

Shapley and Shubik (1972) proves that the core of any assignment game is always nonempty. Among the core allocations of an assignment game, there are two specific extreme core points: the buyers-optimal core allocation $\left(\bar{x}^{A}, \underline{y}^{A}\right)$ where each buyer attains her maximum core payoff and each seller his minimum, and the sellers-optimal core allocation $\left(\underline{x}^{A}, \bar{y}^{A}\right)$ where each seller attains his maximum core payoff and each buyer her minimum.

In Martínez-de-Albéniz et al. (2011) an alternative characterization of the core is given. Let $(\mathcal{F}, \mathcal{W}, A)$ be a square assignment market. Then the core is described as those imputations $(x, y) \in \mathbb{R}_{+}^{m} \times \mathbb{R}_{+}^{m}$ satisfying

$$
x_{i}+y_{j} \leq v_{A}(\mathcal{F} \cup \mathcal{W})-v_{A}\left(\mathcal{F} \cup \mathcal{W} \backslash\left\{f_{i}, w_{j}\right\}\right) \text { for all }\left(f_{i}, w_{j}\right) \in \mathcal{F} \times \mathcal{W}
$$

Moreover, given two square assignment markets $(\mathcal{F}, \mathcal{W}, A)$ and $(\mathcal{F}, \mathcal{W}, B)$, their cores coincide $C\left(v_{A}\right)=C\left(v_{B}\right)$ if and only if

$$
v_{A}\left(\mathcal{F} \cup \mathcal{W} \backslash\left\{f_{i}, w_{j}\right\}\right)=v_{B}\left(\mathcal{F} \cup \mathcal{W} \backslash\left\{f_{i}, w_{j}\right\}\right), \text { for all }\left(f_{i}, w_{j}\right) \in \mathcal{F} \times \mathcal{W} \text {. }
$$

\section{Assortative assignments. Extreme core allocations}

Assortative assignment markets were introduced by Becker (1973). These markets model special bilateral assignment problems where agents on each side can be ordered by some trait with the consequence that the mating of the likes or a positive assortative mating will form. Assortativity is modeled 
by two monotonic effects with respect to the traits: the output effect and the increment of output effect.

An assignment market $(\mathcal{F}, \mathcal{W}, A)$ is an assortative market if it satisfies: a) supermodularity $^{4}$ (the main diagonal is optimal in any $2 \times 2$ submarket):

$$
a_{i l}+a_{k j} \leq a_{i j}+a_{k l} \quad \text { for all } 1 \leq i<k \leq m \text {, and } 1 \leq j<l \leq n .
$$

b) monotonicity (non-decreasing rows and columns):

$$
a_{i j} \leq a_{k l} \text { for all } 1 \leq i \leq k \leq m, \text { and } 1 \leq j \leq l \leq n \text {. }
$$

Matrix $A$ is called assortative.

Only adjacent rows and adjacent columns are needed to check the supermodularity condition.

The class of assortative matrices is large enough. In fact, they form a full-dimensional convex cone in $\mathrm{M}_{m \times n}^{+}$(see Eriksson et al., 2000).

Let $(\mathcal{F}, \mathcal{W}, A)$ be an assortative market with $m \leq n$. From the supermodularity condition at least one optimal matching $\mu \in \mathcal{M}(\mathcal{F}, \mathcal{W})$ is monotone, i.e.

for all $f_{i_{1}}, f_{i_{2}} \in \mathcal{F}$ and $w_{j_{1}}=\mu\left(f_{i_{1}}\right), w_{j_{2}}=\mu\left(f_{i_{2}}\right) \quad$ if $i_{1}<i_{2}$ then $j_{1}<j_{2}$.

When the assortative assignment market is square, that is $m=n$, there is only one monotone matching which is placed in the main diagonal i.e. $\mu\left(f_{i}\right)=w_{i}$ for $i=1,2, \ldots, m$. This is, by the previous observation, optimal (maybe not unique).

\footnotetext{
${ }^{4}$ Notice that this condition implies that matrix entries as function of the indices is supermodular in the lattice $\{1, \ldots, m\} \times\{1, \ldots, n\}$ with the usual order. When the reverse inequalities hold, it is called submodularity.
} 
We will concentrate in the square case since any non-square assortative matrix with $m<n$ could be analyzed by adding null rows at the beginning of the matrix. In this way we preserve supermodularity and the monotonicity conditions.

Now we summarize some important and known results for any square assortative assignment game $\left(\mathcal{F} \cup \mathcal{W}, v_{A}\right)$ with $A \in \mathrm{M}_{m}^{+}$.

(a) The main diagonal of the assignment matrix $A$ is an optimal matching (maybe not unique).

(b) An allocation $(x, y) \in \mathbb{R}_{+}^{m} \times \mathbb{R}_{+}^{m}$ belongs to the core $C\left(v_{A}\right)$ if and only if

$$
\begin{aligned}
& x_{i}+y_{i}=a_{i i} \quad \text { for all } i=1,2, \ldots, m, \\
& x_{i}+y_{i+1} \geq a_{i+1} \quad \text { for all } i=1,2, \ldots, m-1, \\
& x_{i+1}+y_{i} \geq a_{i+1 i} \quad \text { for all } i=1,2, \ldots, m-1 \text {. }
\end{aligned}
$$

(c) At any core allocation $(x, y) \in C\left(v_{A}\right)$ we have

$$
\begin{aligned}
& 0 \leq x_{1} \leq x_{2} \leq \ldots \leq x_{m}, \\
& 0 \leq y_{1} \leq y_{2} \leq \ldots \leq y_{m}
\end{aligned}
$$

Items (a) and (b) depend only on the supermodularity condition of the assignment matrix, and a proof can be found in Martínez-de-Albéniz and Rafels (2014). Item (c) depends only on the monotonicity and the fact that we have an optimal matching in the main diagonal. It is known for assortative matrices (see Eriksson et al., 2000).

Now we give a new and simple procedure to obtain all the extreme core points. To this end, for notational convenience we introduce, for any square assortative assignment market $(\mathcal{F}, \mathcal{W}, A)$ firm 0 and worker 0 and denote $a_{00}=a_{01}=a_{10}=0$. 
A central path (a path) $p$ is a sequence of different places or positions in $(\mathcal{F} \cup\{0\}) \times(\mathcal{W} \cup\{0\})$

$p=\left((0,0),\left(i_{1}, j_{1}\right),(1,1),\left(i_{2}, j_{2}\right),(2,2), \ldots,(m-1, m-1),\left(i_{m}, j_{m}\right),(m, m)\right)$,

where $(k-1, k-1) \leq\left(i_{k}, j_{k}\right) \leq(k, k), i_{k} \neq j_{k}$, for $k=1,2, \ldots, m$.

Notice that for any central path $p$ and any $k=1,2, \ldots, m$, either $(k-1, k)$ or $(k, k-1)$ belongs to $p$ but not both. Therefore there are $2^{m}$ different paths. The set of all paths is denoted by $\mathcal{P}^{m}$.

A central path connects the initial position $(0,0)$ with the last one $(m, m)$ within the central strip. There are two important central paths, the one through the upper part of the strip:

$$
p^{*}=((0,0),(0,1),(1,1),(1,2), \ldots,(m-1, m-1),(m-1, m),(m, m)),
$$

and its symmetric path:

$$
p_{*}=((0,0),(1,0),(1,1),(2,1), \ldots,(m-1, m-1),(m, m-1),(m, m)) .
$$

For each path $p \in \mathcal{P}^{m}$ we associate an allocation vector, the $p$-vector $\left(x^{p}, y^{p}\right) \in \mathbb{R}^{m} \times \mathbb{R}^{m}$, by solving the linear equations given by all the places of the selected path

$$
\begin{aligned}
& x_{k}^{p}+y_{k}^{p}=a_{k k} \quad \text { for } k=1, \ldots, m \text {, and } \\
& x_{i_{k}}^{p}+y_{j_{k}}^{p}=a_{i_{k} j_{k}} \quad \text { for } \quad k=1, \ldots, m \text {, }
\end{aligned}
$$

where we take for notational convenience $x_{0}^{p}=y_{0}^{p}=0$.

For each path $p$ the above linear system has a unique solution, which satisfies $x_{k}^{p} \geq 0$ and $y_{k}^{p} \geq 0$ for $k=1, \ldots, m,\left(x^{p}, y^{p}\right) \in C\left(v_{A}\right)$, and in fact it is an extreme core point. We prove it by induction over $m$. 
Firstly notice that if $m=1$ there are only two different paths which vector is either $\left(a_{11}, 0\right)$ or $\left(0, a_{11}\right)$. Assume that the solution is unique up to $k, k>1$, and without loss of generality assume path $p$ contains $(k, k+1)$. Then by (10) and (11) we have

$$
\begin{aligned}
x_{k}^{p}+y_{k}^{p} & =a_{k k}, \\
x_{k}^{p}+y_{k+1}^{p} & =a_{k k+1}, \\
x_{k+1}^{p}+y_{k+1}^{p} & =a_{k+1 k+1} .
\end{aligned}
$$

Simple manipulations yield, where we use the definition of assortative matrix, see (3) and (4),

$$
\begin{aligned}
y_{k+1}^{p} & =y_{k}^{p}+\left[a_{k k+1}-a_{k k}\right] \geq y_{k}^{p} \geq 0, \\
x_{k+1}^{p} & =x_{k}^{p}+\left[a_{k+1 k+1}-a_{k k+1}\right] \geq x_{k}^{p} \geq 0, \\
x_{k+1}^{p}+y_{k}^{p} & =a_{k+1 k+1}+a_{k k}-a_{k k+1} \geq a_{k+1 k} .
\end{aligned}
$$

From here we obtain the uniqueness of the solution $\left(x^{p}, y^{p}\right)$ and the fact that $\left(x^{p}, y^{p}\right) \in C\left(v_{A}\right)$.

Moreover $\left(x^{p}, y^{p}\right)$ is an extreme core point. To see it, just notice that if it were the midpoint of two other core points, these points must satisfy with equality all entries of path $p$. By uniqueness of the solution, they coincide with $\left(x^{p}, y^{p}\right)$.

We know that each central path gives an extreme core point. Let us write $\mathcal{E} x t\left(C\left(v_{A}\right)\right)$ the set of all extreme core points. We prove next that any extreme core point is linked to a central path, that is, there is a correspondence between paths and extreme core points. This is the following theorem. All the omitted proofs are in the Appendix. 
Theorem 3.1. Let $(\mathcal{F}, \mathcal{W}, A)$ be a square assortative assignment market with $A \in \mathrm{M}_{m}^{+}$. Then

$$
\mathcal{E} x t\left(C\left(v_{A}\right)\right)=\left\{\left(x^{p}, y^{p}\right)\right\}_{p \in \mathcal{P}^{m}}
$$

From the previous theorem there are at most $2^{m}$ different extreme core points. As the reader may suspect, different paths can give the same extreme core point. Therefore to analyze this issue we introduce some additional notation.

Given an square assortative matrix $A$, we define its principal elements

$$
\begin{aligned}
& d_{1}^{A}=a_{11} \geq 0 \\
& d_{k}^{A}=a_{k-1 k-1}+a_{k k}-a_{k-1 k}-a_{k k-1} \geq 0 \text { for } k=2, \ldots, m .
\end{aligned}
$$

Notice that each $d_{k}^{A}, k=2, \ldots, m$, corresponds to a consecutive $2 \times 2$ submarket centered at the main diagonal. The number of extreme core points is strongly related to the number of nonzero principal elements, $n_{A}$, that is

$$
n_{A}=\left|\left\{k \in\{1,2, \ldots, m\} \mid d_{k}^{A} \neq 0\right\}\right| .
$$

Then we have the following theorem.

Theorem 3.2. Let $(\mathcal{F}, \mathcal{W}, A)$ be a square assortative assignment market with $A \in \mathrm{M}_{m}^{+}$. Then

$$
\left|\mathcal{E} x t\left(C\left(v_{A}\right)\right)\right|=2^{n_{A}} .
$$

The proof is a direct consequence of the next lemma.

In the next lemma we analyze what happens with the allocation associated to a path when we move one and only one of its positions. In this way we realize whether this change implies an actual change in the allocation. This fact allows to count the number of extreme core points. 
Lemma 3.1 (Switching lemma). Let $(\mathcal{F}, \mathcal{W}, A)$ be a square assortative assignment market with $A \in \mathrm{M}_{m}^{+}$and let $p \in \mathcal{P}^{m}$ be a central path through $\left(i_{k^{*}}, j_{k^{*}}\right)=\left(k^{*}, k^{*}-1\right)$ for some $k^{*} \in\{1, \ldots, m\}$. Let $p^{\prime} \in \mathcal{P}^{m}$ be the same path as $p$ except we have switched $\left(i_{k^{*}}, j_{k^{*}}\right)$ position to $\left(k^{*}-1, k^{*}\right)$. Then we have

$$
\begin{array}{lll}
x_{k}^{p^{\prime}}=x_{k}^{p} & \text { and } \quad y_{k}^{p^{\prime}}=y_{k}^{p} & \text { for } 1 \leq k \leq k^{*}-1, \\
x_{k}^{p^{\prime}}=x_{k}^{p}+d_{k^{*}}^{A} & \text { and } \quad y_{k}^{p^{\prime}}=y_{k}^{p}-d_{k^{*}}^{A} & \text { for } \quad k^{*} \leq k \leq m,
\end{array}
$$

where $\left(x^{p}, y^{p}\right)$ and $\left(x^{p^{\prime}}, y^{p^{\prime}}\right)$ are defined in (10) and (11), and $d_{k^{*}}^{A}$ in (12) and (13).

Proof. We have to prove that the allocation defined in (14) satisfies equalities of path $p^{\prime}$. Since $\left(x^{p}, y^{p}\right)$ is associated with path $p$, the only equality we have to check is the corresponding to the position $\left(k^{*}-1, k^{*}\right)$. Then for $k^{*} \neq 1$,

$$
\begin{aligned}
x_{k^{*}-1}^{p}+\left(y_{k^{*}}^{p}-d_{k^{*}}^{A}\right) & =\left[a_{k^{*}-1 k^{*}-1}-y_{k^{*}-1}^{p}\right]+\left[a_{k^{*} k^{*}}-x_{k^{*}}^{p}\right]-d_{k^{*}}^{A}= \\
& =a_{k^{*}-1 k^{*}}+a_{k^{*} k^{*}-1}-y_{k^{*}-1}^{p}-x_{k^{*}}^{p}=a_{k^{*}-1 k^{*}} .
\end{aligned}
$$

For $k^{*}=1$, recall $y_{1}^{p}=a_{11}$ and $x_{0}^{p}+\left(y_{1}^{p}-d_{1}^{A}\right)=0=a_{01}$, finishing the proof.

It rests to analyze the proof of Theorem 3.2. Notice that only when in the switching lemma, Lemma 3.1, $d_{k^{*}}^{A}=0$ then the extreme core points corresponding to $p$ and $p^{\prime}$ coincide. Therefore each time a principal element vanishes, the initial potential number of $2^{m}$ extremes is divided by 2 , which concludes the proof.

This switching lemma indicates that whenever we raise up a path, firms' sector is benefited, and consequently, workers' sector is harmed. Recall that $d_{k}^{A} \geq 0$ for all $k \in\{1, \ldots, m\}$. Clearly whenever a path is modified 
downward, workers' sector is benefited. Then as a direct consequence of the switching lemma we have $\left(x^{p^{*}}, y^{p^{*}}\right)=\left(\bar{x}^{A}, \underline{y}^{A}\right)$, where $p^{*}$ is the path given in (8), and path given in (9) is the path for the workers-optimal core allocation, i.e. $\left(x^{p_{*}}, y^{p_{*}}\right)=\left(\underline{x}^{A}, \bar{y}^{A}\right)$.

\section{The solution}

We want to show that a specific point solution has outstanding properties. We define this solution and prove that it is a core point.

Definition 4.1. Let $(\mathcal{F}, \mathcal{W}, A)$ be a square assortative assignment market with $A \in \mathrm{M}_{m}^{+}$. The solution is defined by $\left(x^{*}, y^{*}\right) \in \mathbb{R}_{+}^{m} \times \mathbb{R}_{+}^{m}$ where for $i=1,2, \ldots, m$,

$$
\begin{aligned}
& x_{i}^{*}=\frac{1}{2} a_{i i}+\frac{1}{2} \sum_{k=1}^{i-1} a_{k+1 k}-\frac{1}{2} \sum_{k=1}^{i-1} a_{k k+1}, \\
& y_{i}^{*}=\frac{1}{2} a_{i i}-\frac{1}{2} \sum_{k=1}^{i-1} a_{k+1 k}+\frac{1}{2} \sum_{k=1}^{i-1} a_{k k+1} .
\end{aligned}
$$

We prove now that this solution is a core element, i.e. $\left(x^{*}, y^{*}\right) \in C\left(v_{A}\right)$. To see that all components are non-negative, just observe that for $i=$ $1, \ldots, m-1$

$$
\begin{aligned}
x_{i+1}^{*}-x_{i}^{*} & =\frac{1}{2}\left[a_{i+1 i+1}+a_{i+1 i}-a_{i i+1}-a_{i i}\right] \\
& =\frac{1}{2}\left[a_{i+1 i+1}+a_{i i}-a_{i+1 i}-a_{i i+1}\right]+\left[a_{i+1 i}-a_{i i}\right] \geq 0,
\end{aligned}
$$

using the supermodularity and monotonicity of matrix $A$, see (3) and (4). Moreover $x_{1}^{*}=\frac{1}{2} a_{11} \geq 0$. We have proved

$$
x_{m}^{*} \geq x_{m-1}^{*} \geq \cdots \geq x_{2}^{*} \geq x_{1}^{*} \geq 0 .
$$

Similarly,

$$
y_{m}^{*} \geq y_{m-1}^{*} \geq \cdots \geq y_{2}^{*} \geq y_{1}^{*} \geq 0 .
$$


Trivially $x_{i}^{*}+y_{i}^{*}=a_{i i}$ for $i=1, \ldots, m$. Finally, for $i=1, \ldots, m-1$, we have $x_{i}^{*}+y_{i+1}^{*} \geq a_{i i+1}$ and $x_{i+1}^{*}+y_{i}^{*} \geq a_{i+1}$. We prove the first ones and the others are proved similarly. For $i=1, \ldots, m-1$, we have

$$
x_{i}^{*}+y_{i+1}^{*}=\frac{1}{2}\left[a_{i+1 i+1}+a_{i i}-a_{i+1 i}-a_{i i+1}\right]+a_{i i+1} \geq a_{i i+1},
$$

where we have used the supermodularity condition. Now using (5)-(7) we obtain $\left(x^{*}, y^{*}\right) \in C\left(v_{A}\right)$.

Now we define the median stable utility solution introduced by Schwarz and Yenmez (2011).

Definition 4.2 (Schwarz and Yenmez, 2011). Let $(\mathcal{F}, \mathcal{W}, A)$ be an square assignment market with $A \in \mathrm{M}_{m}^{+}$. The median stable utility solution (MSUS) is the unique core allocation $v_{\text {median }}(A)=(\widetilde{x}, \widetilde{y})$ such that, for all $f_{i} \in \mathcal{F}$ and for all $w_{j} \in \mathcal{W}$,

$$
\begin{aligned}
& \lambda_{r}\left(\left\{(x, y) \in C\left(v_{A}\right) \mid x_{i} \geq \widetilde{x}_{i}\right\}\right)=\lambda_{r}\left(\left\{(x, y) \in C\left(v_{A}\right) \mid x_{i} \leq \widetilde{x}_{i}\right\}\right), \\
& \lambda_{r}\left(\left\{(x, y) \in C\left(v_{A}\right) \mid y_{j} \geq \widetilde{y}_{j}\right\}\right)=\lambda_{r}\left(\left\{(x, y) \in C\left(v_{A}\right) \mid y_{j} \leq \widetilde{y}_{j}\right\}\right),
\end{aligned}
$$

where $\lambda_{r}$ is the Lebesgue measure in $\mathbb{R}^{r}$, with $r=\operatorname{dim} C\left(v_{A}\right)$, see Núñez and Rafels (2008).

We prove next that our solution coincides with the median stable utility solution and the midpoint between the buyers-optimal and the sellersoptimal core allocations. This last coincidence is stated in Schwarz and Yenmez (2011). Moreover, we prove later that it coincides with the nucleolus of the assignment game. Therefore we provide a nice formula for the nucleolus using only the matrix entries to compute it.

Theorem 4.1. Let $(\mathcal{F}, \mathcal{W}, A)$ be a square assortative assignment market with $A \in \mathrm{M}_{m}^{+}$. Then the following statements are equivalent: 
1. $(u, v)=\frac{1}{2}\left[\left(x^{p^{*}}, y^{p^{*}}\right)+\left(x^{p_{*}}, y^{p_{*}}\right)\right]$, where $p^{*}$ and $p_{*}$ are given by (8) and $(9)$

2. $(u, v)=\left(x^{*}, y^{*}\right)$, where $\left(x^{*}, y^{*}\right)$ is given in Definition 4.1,

3. $(u, v)=(\widetilde{x}, \widetilde{y})$ where $(\widetilde{x}, \widetilde{y})$ is given in Definition 4.2.

Proof. In order to prove the theorem we only need to check that $\left(x^{*}, y^{*}\right)=$ $\frac{1}{2}\left[\left(x^{p^{*}}, y^{p^{*}}\right)+\left(x^{p_{*}}, y^{p_{*}}\right)\right]$, since by Schwarz and Yenmez $(2011) v_{\text {median }}(A)=$ $(\widetilde{x}, \widetilde{y})=\frac{1}{2}\left[\left(x^{p^{*}}, y^{p^{*}}\right)+\left(x^{p_{*}}, y^{p_{*}}\right)\right]$ for any square assortative assignment market.

Clearly $u_{1}=v_{1}=\frac{1}{2} a_{11}$ and for $k=1, \ldots, m$, we have $u_{k}+v_{k}=a_{k k}$. Notice that for all $k \in\{2, \ldots, m\}$ position $(k-1, k)$ belongs to path $p^{*}$ and position $(k, k-1)$ to path $p_{*}$. Then we have, for $k=2, \ldots, m$.

$$
\begin{aligned}
u_{k-1}+v_{k} & =\frac{1}{2}\left[\left(x_{k-1}^{p^{*}}+x_{k-1}^{p_{*}}\right)+\left(y_{k}^{p^{*}}+y_{k}^{p_{*}}\right)\right] \\
& =\frac{1}{2}\left[a_{k-1 k}+x_{k-1}^{p_{*}}+y_{k}^{p_{*}}\right] \\
& =\frac{1}{2}\left[a_{k-1 k}+a_{k-1 k-1}+a_{k k}-a_{k k-1}\right] \\
& =a_{k-1 k}+\frac{1}{2} d_{k}^{A}
\end{aligned}
$$

Solving the above linear equations we easily obtain

$$
\begin{array}{ll}
u_{i}=\frac{1}{2}\left[a_{i i}+\sum_{k=1}^{i-1} a_{k+1 k}-\sum_{k=1}^{i-1} a_{k k+1}\right] & \text { for } i=1, \ldots, m, \\
v_{j}=\frac{1}{2}\left[a_{j j}-\sum_{k=1}^{j-1} a_{k+1 k}+\sum_{k=1}^{j-1} a_{k k+1}\right] & \text { for } j=1, \ldots, m .
\end{array}
$$

Now we turn to the nucleolus of the square assortative assignment game. Among other solutions, the nucleolus (Schmeidler, 1969) is a "fair" solution in the general context of cooperative games. For balanced games, it 
is the unique core-selection that lexicographically minimizes the excesses ${ }^{5}$ arranged in a nondecreasing way.

Here, we use the characterization of the nucleolus of a square assignment game of Llerena and Núñez (2011). Given any square assignment game $\left(\mathcal{F} \cup \mathcal{W}, v_{A}\right)$ and two arbitrary coalitions $\emptyset \neq S \subseteq \mathcal{F}$ and $\emptyset \neq T \subseteq \mathcal{W}$, we define

$$
\begin{aligned}
& \delta_{S, T}^{A}(x, y):=\min _{i \in S, j \in \mathcal{W} \backslash T}\left\{x_{i}, x_{i}+y_{j}-a_{i j}\right\}, \\
& \delta_{T, S}^{A}(x, y):=\min _{j \in T, i \in \mathcal{F} \backslash S}\left\{y_{j}, x_{i}+y_{j}-a_{i j}\right\},
\end{aligned}
$$

for any core allocation $(x, y) \in C\left(v_{A}\right)$.

Llerena and Núñez (2011) prove that the nucleolus of a square assignment game $\nu\left(v_{A}\right)$ is characterized as the unique core allocation $(x, y) \in C\left(v_{A}\right)$ such that for some optimal matching $\mu \in \mathcal{M}_{A}^{*}(\mathcal{F}, \mathcal{W})$

$$
\delta_{S, \mu(S)}^{A}(x, y)=\delta_{\mu(S), S}^{A}(x, y), \text { for any } \emptyset \neq S \subseteq \mathcal{F}
$$

Now we are in the position to prove that our solution coincides with the nucleolus of the assortative assignment game. Its rather technical proof can be found in the Appendix.

Theorem 4.2. Let $(\mathcal{F}, \mathcal{W}, A)$ be a square assortative assignment market, and $\left(\mathcal{F} \cup \mathcal{W}, v_{A}\right)$ be its associated cooperative game. Then, the solution defined in Definition 4.1 coincides with the nucleolus of the game, i.e.

$$
\left(x^{*}, y^{*}\right)=\nu\left(v_{A}\right)
$$

\footnotetext{
${ }^{5}$ Given a coalition $S \subseteq N$, and an allocation $x \in \mathbb{R}^{N}$ the excess of a coalition is defined as $e(S, x):=v(S)-\sum_{i \in S} x_{i}$. Note they can be considered as complaints.
} 


\section{The solution and the Shapley value}

The most popular single point solution for cooperative games is the Shapley value (Shapley, 1953). This solution assigns a vector for any cooperative game, and it is based on marginalistic considerations. It can be regarded as an ex-ante evaluation of the value (power) of any player in the game. It is defined as the mean of all marginal worth vectors and a specific formula can be derived. For any cooperative game $(N, v)$ the Shapley value $S h(v)$ is defined for all $i \in N$ as

$$
S h_{i}(v)=\sum_{S \subseteq N \backslash\{i\}} \frac{s !(n-s-1) !}{n !}[v(S \cup\{i\})-v(S)],
$$

where $|S|=s$.

It is well-known that the Shapley value is usually outside the core for assignment games. Hoffmann and Sudhölter (2007) study the Shapley value for assignment games and prove that for all exact assignment games the Shapley value belongs to the core. They prove that this is a sufficient but not a necessary condition with a thorough study of $2 \times 2$ assignment games. Exact assignment games have been studied by Solymosi and Raghavan (2001), where they derive two conditions: dominant diagonal and doubly-dominant diagonal.

Now we investigate the relationship between the Shapley value and our solution, and show that for square assortative assignment games, both solutions coincide only for some specific assortative matrices. This is our next theorem.

Theorem 5.1. Let $(\mathcal{F}, \mathcal{W}, A)$ be a square assortative assignment market, and $\left(\mathcal{F} \cup \mathcal{W}, v_{A}\right)$ its associated cooperative game. The following statements are equivalent: 
1. The solution in Definition 4.1 and the Shapley value coincide,

$$
\left(x^{*}, y^{*}\right)=\operatorname{Sh}\left(v_{A}\right)
$$

2. The Shapley value belongs to the core, $\operatorname{Sh}\left(v_{A}\right) \in C\left(v_{A}\right)$.

3. Matrix $A$ is as follows:

$$
A=\left(\begin{array}{cccc}
\alpha_{1} & \alpha_{1} & \ldots & \alpha_{1} \\
\cline { 2 - 4 } \alpha_{1} & \alpha_{2} & \ldots & \alpha_{2} \\
\vdots & \vdots & \ddots & \vdots \\
\alpha_{1} & \alpha_{2} & \ldots & \alpha_{m}
\end{array}\right), \quad \text { with } \quad 0 \leq \alpha_{1} \leq \alpha_{2} \leq \ldots \leq \alpha_{m}
$$

Roughly speaking, this result shows that the Shapley value belongs to the core of an assortative assignment game only when the dominant diagonal property holds. Combined with the monotonicity, we obtain the special structure of the matrix.

\section{Extensions}

Now we introduce some extensions of the assortative market notion. The first one contributes to relax the monotonicity condition (4). This possibility was also pointed out in Sherstyuk (1999). We name this concept weak-assortative and basically, apart from the supermodularity conditions, we request the monotonicity only on the central strip of the matrix.

Given an assignment market $(\mathcal{F}, \mathcal{W}, A)$ where $A \in \mathrm{M}_{m}^{+}$we say it is a weak-assortative market if it satisfies supermodularity condition (3) and an alternative condition to (4), that is

b') central strip monotonicity (entries of the central strip satisfy mono- 
tonicity):

$$
\begin{gathered}
a_{i j} \leq a_{k l} \text { for all } 1 \leq i \leq k \leq m, \text { and } 1 \leq j \leq l \leq m \\
\text { with }|i-j| \leq 1 \text { and }|k-l| \leq 1
\end{gathered}
$$

As the name suggests, weak-assortative is a proper extension of the assortative class of assignment markets. Nevertheless there is a kind of reverse implication. The core of any weak-assortative market equals the core of an assortative one. This is the essence to transfer the properties from assortative markets to weak-assortative ones. This result is constructive.

We introduce for any square matrix $A \in \mathrm{M}_{m}^{+}$the matrix $\widetilde{A} \in \mathrm{M}_{m}$ defined by:

$$
\tilde{a}_{i j}= \begin{cases}\sum_{k=i}^{j-1} a_{k k+1}-\sum_{k=i+1}^{j-1} a_{k k} & \text { for } 1 \leq i<j \leq m, \\ a_{i i} & \text { for } 1 \leq i=j \leq m, \\ \sum_{k=j}^{i-1} a_{k+1 k}-\sum_{k=j+1}^{i-1} a_{k k} & \text { for } 1 \leq j<i \leq m,\end{cases}
$$

where the summation over an empty set of indices is zero. Notice that in general, entries $\widetilde{a}_{i j}$ may be negative, but if matrix $A$ is weak-assortative, then $\widetilde{A} \in \mathrm{M}_{m}^{+}$, since expression (22) can be written as:

$$
\widetilde{a}_{i j}= \begin{cases}a_{i i+1}+\sum_{k=i+1}^{j-1}\left[a_{k k+1}-a_{k k}\right] \geq 0 & \text { for } 1 \leq i<j \leq m, \\ a_{i i} & \text { for } 1 \leq i=j \leq m, \\ a_{j+1 j}+\sum_{k=j+1}^{i-1}\left[a_{k+1 k}-a_{k k}\right] \geq 0 & \text { for } 1 \leq j<i \leq m .\end{cases}
$$

Notice that matrices $A$ and $\widetilde{A}$ have the same central strip.

Theorem 6.1. Let $(\mathcal{F}, \mathcal{W}, A)$ be an square weak-assortative market with $A \in \mathrm{M}_{m}^{+}$. Then there exists an assortative market $(\mathcal{F}, \mathcal{W}, \widetilde{A})$ with $\widetilde{A} \in \mathrm{M}_{m}^{+}$ given by (22) with the same core

$$
C\left(v_{A}\right)=C\left(v_{\widetilde{A}}\right) .
$$


As a consequence of the above theorem, we can apply to any weakassortative matrix, directly from its central strip our previous results for assortative matrices, that is, the extreme core points, our solution, and its coincidence with MSUS, the nucleolus, ${ }^{6}$ etc.

Weak-assortative matrices open the possibility to look for a more general class of assignment matrices that can be solved and their core described using an appropriate assortative matrix. These matrices will be called assortativesolvable.

Given an square assignment market $(\mathcal{F}, \mathcal{W}, A)$ where $A \in \mathrm{M}_{m}^{+}$we say it is assortative-solvable if there exists an square assortative matrix $B \in \mathrm{M}_{m}^{+}$ with the same core, that is $C\left(v_{A}\right)=C\left(v_{B}\right)$.

Weak-assortative matrices are assortative-solvable, but there are another ones, as the next example shows.

Example 6.1. Consider the following square assignment market $(\mathcal{F}, \mathcal{W}, A)$ with matrix

$$
A=\left(\begin{array}{cccc}
\mathbf{2 1} & 25 & 27 & 5 \\
26 & \mathbf{3 0} & 2 & 0 \\
7 & 41 & \mathbf{5 2} & 60 \\
8 & 2 & 53 & \mathbf{7 3}
\end{array}\right)
$$

The assignment matrix $A$ is not assortative, not even supermodular, not even central strip monotone. An optimal matching is in boldface.

\footnotetext{
${ }^{6}$ Núñez (2004) proves that for assignment games, equality of cores implies coincidence of nucleolus.
} 
Matrix

$$
B=\left(\begin{array}{cccc}
\mathbf{2 1} & 25 & 26 & 30 \\
26 & \mathbf{3 0} & 32 & 38 \\
37 & 41 & \mathbf{5 2} & 60 \\
37 & 41.5 & 53 & \mathbf{7 3}
\end{array}\right)
$$

is assortative and defines the same core: $C\left(v_{B}\right)=C\left(v_{A}\right)$. To see this equality of the cores, notice that $v_{A}(\mathcal{F} \cup \mathcal{W})=v_{B}(\mathcal{F} \cup \mathcal{W})=176$ and check the equalities in (2).

As an application we know that $C\left(v_{A}\right)$ has exactly $2^{n_{B}}=8$ extreme core allocations, the firms-optimal and workers-optimal core allocations are

$$
\begin{aligned}
& \left(\bar{x}^{A}, \underline{y}^{A}\right)=(21,26,46,59 ; 0,4,6,14), \\
& \left(\underline{x}^{A}, \bar{y}^{A}\right)=(0,5,16,17 ; 21,25,36,56) .
\end{aligned}
$$

The nucleolus of the assignment game $\left(\mathcal{F} \cup \mathcal{W}, v_{A}\right)$ is just the middle point or the solution of Definition 4.1 applied to matrix $B$ :

$$
\nu\left(v_{A}\right)=\nu\left(v_{B}\right)=(10.5,15.5,31,38 ; 10.5,14.5,21,35) .
$$

Notice that we have to use matrix $B$ to obtain the extreme core allocations or our solution. We cannot apply our methods directly to matrix A, since the central strip changes from matrix $A$ to the assortative matrix $B$.

Two natural questions arise. Firstly how to know whether matrix $A \in$ $\mathrm{M}_{m}^{+}$is assortative-solvable and secondly how to find an associated assortative matrix. First of all, we fix an optimal matching on the main diagonal: the optimal partner of firm $f_{k} \in \mathcal{F}$ is worker $w_{k} \in \mathcal{W}$.

Given a matrix $A \in \mathrm{M}_{m}^{+}$with an optimal matching placed on the main diagonal, we introduce an auxiliary matrix $H(A)=\left(h_{i j}^{A}\right) \in \mathrm{M}_{m}^{+}$defined by

$$
h_{i j}^{A}=v_{A}(\mathcal{F} \cup \mathcal{W})-v_{A}\left(\mathcal{F} \cup \mathcal{W} \backslash\left\{f_{j}, w_{i}\right\}\right), \quad \text { for } i, j=1, \ldots m .
$$


Notice that in (24) we subtract the worth of the market without the partners of firm $f_{i}$ and worker $w_{j}$, that is, we drop out worker $w_{i}$ and firm $f_{j}$. This matrix is the cornerstone for our characterization.

In the above Example 6.1 we can compute matrix $H(A)$ :

$$
H(A)=\left(\begin{array}{llll}
21 & 26 & 46 & 59 \\
25 & 30 & 50 & 63 \\
36 & 41 & 52 & 65 \\
56 & 61 & 72 & 73
\end{array}\right) .
$$

Notice that matrix $H(A)$ is submodular and monotonic ${ }^{7}$. This will be our main result, since both properties for matrix $H(A)$ characterize the assortative-solvability of matrix $A$. Moreover, an assortative matrix $B$ is directly related to $H(A)$ as next theorem shows.

Theorem 6.2. Let $(\mathcal{F}, \mathcal{W}, A)$ be a square assignment market where $A \in \mathrm{M}_{m}^{+}$ and an optimal matching is placed on the main diagonal. Let matrix $H(A)$ be the matrix defined in (24). Then the following statements are equivalent:

1. Matrix $A$ is assortative-solvable.

2. Matrix $H(A)$ is submodular and monotonic.

Moreover, in this case, an assortative matrix $B \in \mathrm{M}_{m}^{+}$with the same core is given by $b_{i j}=a_{i i}+a_{j j}-h_{i j}^{A}$, for all $\left(f_{i}, w_{j}\right) \in \mathcal{F} \times \mathcal{W}$.

Notice that in Example 6.1 matrix $B$ given by Theorem 6.2 does not coincide with the one in the example. This shows that an assortative-solvable matrix does not determine the assortative matrix which corresponds to it.

\footnotetext{
${ }^{7}$ For the definition of submodular, see (3) but reversing the inequalities, and for monotonicity see (4).
} 
One possible extension of the current paper is to many-to-one markets in the tradition of Kelso and Crawford (1982). In this setting, Chen et al. (2016) show the existence of a median stable matching, and this is a line of future research.

[1] Becker, G.S., 1973. A Theory of Marriage: Part I. The Journal of Political Economy 81(4), 813-846.

[2] Chen, P., Egesdal, M., Pycia, M., Yenmez, M.B., 2016. Median stable matchings in two-sided markets. Games and Economic Behavior 97, $64-69$.

[3] Eriksson, K., Karlander, J., Öller, L.-E., 2000. Becker's assortative assignments: stability and fairness. Mathematical Social Sciences 39, $109-118$.

[4] Hamers, H.J.M., Klijn, F., Solymosi, T., Tijs, S.H., Villar, J.P., 2002. Assignment games satisfy the CoMa property. Games and Economic Behavior 38(2), 231-239.

[5] Hoffmann, M., Sudhölter, P., 2007. The Shapley value of exact assignment games. International Journal of Game Theory 35, 557-568.

[6] Kelso, A.S., Crawford, V.P., 1982. Job matching, coalition formation, and gross substitutes. Econometrica 50, 1483-1504.

[7] Llerena, F., Núñez, M., 2011. A geometric characterization of the nucleolus of the assignment game. Economics Bulletin 31(4), 3275-3285.

[8] Martínez-de-Albéniz, F.J., Núñez, M., Rafels, C., 2011. Assignment markets with the same core. Games and Economic Behavior 73(2), 553563. 
[9] Martínez-de-Albéniz, F.J., Rafels, C., 2014. Cooperative assignment games with the inverse Monge property. Discrete Applied Mathematics $162,42-50$.

[10] Núñez, M., 2004. A note on the nucleolus and the kernel of the assignment game. International Journal of Game Theory 33, 55-65.

[11] Núñez, M., Rafels, C., 2008. On the dimension of the core of the assignment game. Games and Economic Behavior 64, 290-302.

[12] Núñez, M., Rafels, C., 2015. A survey on assignment markets. Journal of Dynamics and Games 3\&4, 227-256.

[13] Roth, A., Sotomayor, M., 1990. Two-sided matching. Econometric Society Monographs, 18. Cambridge University Press.

[14] Schmeidler, D., 1969. The nucleolus of a characteristic function game. SIAM Journal of Applied Mathematics 17, 1163-1170.

[15] Schwarz, M., Yenmez, M.B., 2011. Median stable matching for markets with wages. Journal of Economic Theory 146, 619-637.

[16] Shapley, L.S., 1953. A value for n-person games. In: Contributions to the Theory of Games II, vol 28, Annals of Mathematics Studies. A.W. Tucker and H.W. Kuhn, eds., Princeton University Press, Princeton, pp. 307-317.

[17] Shapley, L.S., Shubik, M., 1972. The assignment game I: the core. International Journal of Game Theory 1, 111-130.

[18] Sherstyuk, K., 1999. Multisided matching games with complementarities. International Journal of Game Theory 28, 489-509. 
[19] Solymosi, T., Raghavan, T.E.S., 2001. Assignment games with stable core. International Journal of Game Theory 30, 177-185.

[20] Thompson, G.L., 1981. Auctions and market games. In: Essays in Game Theory and Mathematical Economics in honor of Oskar Morgenstern. R. Aumann et al., eds. Bibliographisches Institute-Wissenschaftsverlag Mannheim, pp. 181-196.

\section{AppendixA. Proofs}

\section{Proof of Theorem 3.1}

Proof. One inclusion has been discussed previously. For the other, suppose $(x, y)$ is an extreme core point but it does not correspond to any path. Since in any extreme core allocation either $x_{1}=0$ or $y_{1}=0$ (see Hamers et al., 2002), then either $x_{0}+y_{1}=a_{01}$ or $x_{1}+y_{0}=a_{10}$ where $x_{0}=y_{0}=0$ by notational convenience. Let $k \in\{1,2, \ldots, m-1\}$ be the first index where

$x_{k}+y_{k+1}>a_{k+1}$ and $x_{k+1}+y_{k}>a_{k+1 k}$. Notice that $x_{k+1}>0$ since if $x_{k+1}=0$ then $x_{k}=0$ and $a_{k k}=x_{k}+y_{k}=x_{k+1}+y_{k}>a_{k+1 k}$ contradicting (4). Similarly $y_{k+1}>0$.

Now take $\varepsilon \in \mathbb{R}$ defined below

$$
\varepsilon=\min \left\{x_{k+1}, y_{k+1}, x_{k+1}+y_{k}-a_{k+1 k}, x_{k}+y_{k+1}-a_{k k+1}\right\}>0,
$$

and define $\left(x^{\prime}, y^{\prime}\right) \in \mathbb{R}_{+}^{\mathcal{F}} \times \mathbb{R}_{+}^{\mathcal{W}}$ by

$$
\begin{array}{r}
x_{t}^{\prime}=x_{t} \text { and } y_{t}^{\prime}=y_{t}, \quad \text { for } t \leq k, \\
x_{t}^{\prime}=x_{t}+\varepsilon \text { and } y_{t}^{\prime}=y_{t}-\varepsilon, \quad \text { for } t>k .
\end{array}
$$


We have $\left(x^{\prime}, y^{\prime}\right) \in C\left(v_{A}\right)$ and $\left(x^{\prime}, y^{\prime}\right) \neq(x, y)$. Indeed it is enough to see that $x_{k}^{\prime}+y_{k+1}^{\prime}=x_{k}+y_{k+1}-\varepsilon \geq a_{k+1}$.

Similarly define $\left(x^{\prime \prime}, y^{\prime \prime}\right) \in \mathbb{R}_{+}^{\mathcal{F}} \times \mathbb{R}_{+}^{\mathcal{W}}$ by

$$
\begin{aligned}
x_{t}^{\prime \prime}=x_{t} \text { and } \quad y_{t}^{\prime \prime}=y_{t}, \quad \text { for } t \leq k, \\
x_{t}^{\prime \prime}=x_{t}-\varepsilon \text { and } y_{t}^{\prime \prime}=y_{t}+\varepsilon, \quad \text { for } t>k .
\end{aligned}
$$

Then $\left(x^{\prime \prime}, y^{\prime \prime}\right) \in C\left(v_{A}\right)$ and $\left(x^{\prime \prime}, y^{\prime \prime}\right) \neq(x, y)$. Moreover $(x, y)$ is the midpoint between $\left(x^{\prime}, y^{\prime}\right)$ and $\left(x^{\prime \prime}, y^{\prime \prime}\right)$, a contradiction with $(x, y)$ being an extreme core point.

\section{Proof of Theorem 4.2}

Proof. We first prove some technical results that the solution $\left(x^{*}, y^{*}\right)$ in Definition 4.1 satisfies.

Notice first that for $j=2, \ldots, m$,

$$
x_{j}^{*}-x_{j-1}^{*}=\frac{1}{2}\left[a_{j j}+a_{j j-1}-a_{j-1 j}-a_{j-1 j-1}\right] .
$$

Lemma A.1 For all $k \in\{2, \ldots, m\}$ we have

$$
\begin{aligned}
& 0 \geq x_{1}^{*}-a_{1 k} \geq x_{2}^{*}-a_{2 k} \geq \ldots \geq x_{k-1}^{*}-a_{k-1 k}, \\
& 0 \geq y_{1}^{*}-a_{k 1} \geq y_{2}^{*}-a_{k 2} \geq \ldots \geq y_{k-1}^{*}-a_{k k-1} .
\end{aligned}
$$

For the first inequality of (A.2), just observe $a_{1 k} \geq a_{11} \geq \frac{1}{2} a_{11}=x_{1}^{*}$. For the rest of inequalities let $j \in\{2, \ldots, k-1\}$ and because of supermodularity, notice $j<k$, we have

$$
\begin{aligned}
& a_{j k}-a_{j-1 k} \geq a_{j j-1}-a_{j-1 j-1}, \\
& a_{j k}-a_{j-1 k} \geq a_{j j}-a_{j-1 j} .
\end{aligned}
$$

Summing up and using (A.1) we get the desired result. Inequalities (A.3) are left for the reader. 
Lemma A.2 For all $k \in\{1, \ldots, m-1\}$ we have

$$
\begin{gathered}
x_{m}^{*}-a_{m k} \geq x_{m-1}^{*}-a_{m-1 k} \geq \ldots \geq x_{k+1}^{*}-a_{k+1 k}, \\
y_{m}^{*}-a_{k m} \geq y_{m-1}^{*}-a_{k m-1} \geq \ldots \geq y_{k+1}^{*}-a_{k k+1} .
\end{gathered}
$$

To prove (A.4), let $j \in\{k+2, \ldots, m\}$ and by supermodularity, notice $j-1>$ $k$, we have

$$
\begin{aligned}
a_{j j}-a_{j-1 j} & \geq a_{j k}-a_{j-1 k} \\
a_{j j-1}-a_{j-1 j-1} & \geq a_{j k}-a_{j-1 k} .
\end{aligned}
$$

Summing up and using (A.1) we get the desired result. The proof of (A.5) is similar.

Finally and from the expression of the solution in Definition 4.1, it is easy to see that for all $k \in\{1, \ldots, m-1\}$ we have

$x_{k+1}^{*}+y_{k}^{*}-a_{k+1 k}=x_{k}^{*}+y_{k+1}^{*}-a_{k k+1}=\frac{1}{2}\left[a_{k+1 k+1}+a_{k k}-a_{k+1 k}-a_{k k+1}\right]$.

Let $f_{r} \in \mathcal{F}$ and denote $R=\left\{f_{1}, \ldots, f_{r}\right\} \subseteq \mathcal{F}$. Let $A_{R}$ be the matrix $A$ restricted to the first $r$ rows and columns. Notice now that the solution for $A_{R},\left(x_{R}^{*}, y_{R}^{*}\right) \in \mathbb{R}_{+}^{r} \times \mathbb{R}_{+}^{r}$ is just the restriction of the solution for $A$, and that $\left(x_{R}^{*}, y_{R}^{*}\right) \in C\left(v_{A_{R}}\right)$.

Now we prove that $\left(x^{*}, y^{*}\right)$ is the nucleolus by induction over $m$. The case $m=1$ is obvious. Assume the statement is true until $r, r \in\{1, \ldots, m-1\}$ and we prove it for $r+1$. Denote by $R 1=\{1, \ldots, r+1\} \subseteq \mathcal{F}$ and $A_{R 1}$ matrix $A$ restricted to the first $r+1$ rows and columns.

Since matrix $A$ is assortative, the main diagonal is an optimal matching $\mu$. Then $S^{\prime}$ stands for $\mu(S)$ and we must check that

$$
\delta_{S, S^{\prime}}^{A_{R 1}}\left(x_{R 1}^{*}, y_{R 1}^{*}\right)=\delta_{S^{\prime}, S}^{A_{R 1}}\left(x_{R 1}^{*}, y_{R 1}^{*}\right), \text { for any } \emptyset \neq S \subseteq R 1
$$


We proceed through cases.

Case 1: $S \subseteq R, S \neq \emptyset$.

$$
\begin{aligned}
\delta_{S, S^{\prime}}^{A_{R 1}}\left(x_{R 1}^{*}, y_{R 1}^{*}\right)= & \min _{i \in S, j \in R 1 \backslash S}\left\{x_{i}^{*}, x_{i}^{*}+y_{j}^{*}-a_{i j}\right\}= \\
& \min _{i \in S}\left\{\delta_{S, S^{\prime}}^{A_{R}}\left(x_{R}^{*}, y_{R}^{*}\right), x_{i}^{*}+y_{r+1}^{*}-a_{i r+1}\right\}= \\
& \min \left\{\delta_{S, S^{\prime}}^{A_{R}}\left(x_{R}^{*}, y_{R}^{*}\right), x_{i_{s}}^{*}+y_{r+1}^{*}-a_{i_{s} r+1}\right\},
\end{aligned}
$$

where $i_{s}=\max \{i \mid i \in S\}$, and we have used (A.2) with $k=r+1$.

In the same way we obtain

$$
\delta_{S^{\prime}, S}^{A_{R 1}}\left(x_{R 1}^{*}, y_{R 1}^{*}\right)=\min \left\{\delta_{S^{\prime}, S}^{A_{R}}\left(x_{R}^{*}, y_{R}^{*}\right), y_{i_{s}}^{*}+x_{r+1}^{*}-a_{r+1 i_{s}}\right\} .
$$

Now we have two possibilities:

(a) $i_{s}=r$. By induction hypothesis $\delta_{S, S^{\prime}}^{A_{R}}\left(x_{R}^{*}, y_{R}^{*}\right)=\delta_{S^{\prime}, S}^{A_{R}}\left(x_{R}^{*}, y_{R}^{*}\right)$ and by (A.6) we have $x_{r}^{*}+y_{r+1}^{*}-a_{r r+1}=y_{r}^{*}+x_{r+1}^{*}-a_{r+1 r}$ and (A.7) holds.

(b) $i_{s}<r$. Then $i_{s}+1 \leq r$, and by (A.5) taking $k=i_{s}$ we have $x_{i_{s}}^{*}+y_{r+1}^{*}-$ $a_{i_{s} r+1} \geq x_{i_{s}}^{*}+y_{i_{s}+1}^{*}-a_{i_{s} i_{s}+1}$. Now notice $i_{s} \in S, i_{s}+1 \notin S$, and then $x_{i_{s}}^{*}+y_{r+1}^{*}-a_{i_{s} r+1} \geq \delta_{S, S^{\prime}}^{A_{R}}\left(x_{R}^{*}, y_{R}^{*}\right)$. In this case $\delta_{S, S^{\prime}}^{A_{R 1}}\left(x_{R 1}^{*}, y_{R 1}^{*}\right)=$ $\delta_{S, S^{\prime}}^{A_{R}}\left(x_{R}^{*}, y_{R}^{*}\right)$ and similarly $\delta_{S^{\prime}, S}^{A_{R 1}}\left(x_{R 1}^{*}, y_{R 1}^{*}\right)=\delta_{S^{\prime}, S}^{A_{R}}\left(x_{R}^{*}, y_{R}^{*}\right)$, and by the induction hypothesis (A.7) holds.

Case 2: $S=\{r+1\}$.

$$
\begin{aligned}
\delta_{S, S^{\prime}}^{A_{R 1}}\left(x_{R 1}^{*}, y_{R 1}^{*}\right)= & \min _{j \in R}\left\{x_{r+1}^{*}, x_{r+1}^{*}+y_{j}^{*}-a_{r+1 j}\right\}= \\
& \min \left\{x_{r+1}^{*}, x_{r+1}^{*}+y_{r}^{*}-a_{r+1 r}\right\}= \\
& x_{r+1}^{*}+y_{r}^{*}-a_{r+1 r},
\end{aligned}
$$

where we have used (A.3) taking $k=r+1$ and $0 \geq y_{1}^{*}-a_{r+11} \geq y_{r}^{*}-a_{r+1 r}$. 
Similarly,

$$
\delta_{S^{\prime}, S}^{A_{R 1}}\left(x_{R 1}^{*}, y_{R 1}^{*}\right)=x_{r}^{*}+y_{r+1}^{*}-a_{r r+1} .
$$

Then (A.7) holds by (A.6).

Lastly Case 3: $S=\widetilde{S} \cup\{r+1\}, \widetilde{S} \subseteq R, \widetilde{S} \neq \emptyset$.

$$
\begin{aligned}
\delta_{S, S^{\prime}}^{A_{R 1}}\left(x_{R 1}^{*}, y_{R 1}^{*}\right)= & \min _{i \in S, j \in R 1 \backslash S}\left\{x_{i}^{*}, x_{i}^{*}+y_{j}^{*}-a_{i j}\right\}= \\
& \min _{j \in R \backslash \widetilde{S}}\left\{\delta_{\widetilde{S}, \widetilde{S}^{\prime}}^{A_{R}}\left(x_{R}^{*}, y_{R}^{*}\right), x_{r+1}^{*}, x_{r+1}^{*}+y_{j}^{*}-a_{r+1 j}\right\}= \\
& \min \left\{\delta_{\widetilde{S}, \widetilde{S}^{\prime}}^{A_{R}}\left(x_{R}^{*}, y_{R}^{*}\right), x_{r+1}^{*}+y_{j_{t}}^{*}-a_{r+1 j t}\right\},
\end{aligned}
$$

where we have noticed first that $R 1 \backslash S=R \backslash \widetilde{S}$; we have $x_{r+1}^{*} \geq x_{r}^{*} \geq$ $\delta_{\widetilde{S}, \widetilde{S}^{\prime}}^{A_{R}}\left(x_{R}^{*}, y_{R}^{*}\right)$ and we have used (A.3) with $k=r+1$ and $j_{t}=\max \{j \mid j \in R \backslash \widetilde{S}\}$.

In the same way we obtain

$$
\delta_{S^{\prime}, S}^{A_{R 1}}\left(x_{R 1}^{*}, y_{R 1}^{*}\right)=\min \left\{\delta_{\widetilde{S}^{\prime}, \widetilde{S}}^{A_{R}}\left(x_{R}^{*}, y_{R}^{*}\right), y_{r+1}^{*}+x_{j_{t}}^{*}-a_{j_{t} r+1}\right\}
$$

Now we have two possibilities:

(a) $j_{t}=r$. By induction hypothesis $\delta_{\widetilde{S}, \widetilde{S}^{\prime}}^{A_{R}}\left(x_{R}^{*}, y_{R}^{*}\right)=\delta_{\widetilde{S}^{\prime}, \widetilde{S}^{\prime}}^{A_{R}}\left(x_{R}^{*}, y_{R}^{*}\right)$ and by (A.6) we have $x_{r}^{*}+y_{r+1}^{*}-a_{r r+1}=y_{r}^{*}+x_{r+1}^{*}-a_{r+1 r}$ and (A.7) holds.

(b) $j_{t}<r$. Then $j_{t}+1 \leq r$, and by (A.4) taking $k=j_{t}$ we have $x_{r+1}^{*}+$ $y_{j_{t}}^{*}-a_{r+1 j_{t}} \geq x_{j_{t}+1}^{*}+y_{j_{t}}^{*}-a_{j_{t}+1 j_{t}}$. Now notice $j_{t} \in R \backslash \widetilde{S}, j_{t}+$ $1 \notin R \backslash \widetilde{S}$, and then $x_{r+1}^{*}+y_{j_{t}}^{*}-a_{r+1 j_{t}} \geq \delta_{\widetilde{S}, \widetilde{S}^{\prime}}^{A_{R}}\left(x_{R}^{*}, y_{R}^{*}\right)$. In this case $\delta_{S, S^{\prime}}^{A_{R 1}}\left(x_{R 1}^{*}, y_{R 1}^{*}\right)=\delta_{\widetilde{S}, \widetilde{S}^{\prime}}^{A_{R}}\left(x_{R}^{*}, y_{R}^{*}\right)$ and similarly $\delta_{S^{\prime}, S}^{A_{R 1}}\left(x_{R 1}^{*}, y_{R 1}^{*}\right)=$ $\delta_{\widetilde{S}^{\prime}, \widetilde{S}}^{A_{R}}\left(x_{R}^{*}, y_{R}^{*}\right)$. Then (A.7) holds.

This finishes the proof.

\section{Proof of Theorem 5.1}


Proof. 1. $\longrightarrow 2$. Immediate.

2. $\longrightarrow 3$. First, let $\left(\mathcal{F} \cup \mathcal{W}, v_{A}\right)$ be an square assignment game with $A \in$ $\mathrm{M}_{m}^{+}$. Now we relate the Shapley value of this assignment game with the Shapley value of the game when we add the same constant to all matrix entries. This constant can be positive or negative, provided we stay into $\mathrm{M}_{m}^{+}$. Then, from Hoffmann and Sudhölter (2007), for any $t \geq-\min _{\left(f_{i}, w_{j}\right) \in \mathcal{F} \times \mathcal{W}}\left\{a_{i j}\right\}$ define matrix $A^{t}$ by $a_{i j}^{t}=a_{i j}+t$, that is $A^{t}=A+t U$ with matrix $U \in \mathrm{M}_{m}^{+}$ a matrix of ones. Now we have

$$
\begin{aligned}
& S h_{i}\left(v_{A^{t}}\right)=\frac{t}{2}+S h_{i}\left(v_{A}\right), \text { for all } f_{i} \in \mathcal{F}, \\
& S h_{j}\left(v_{A^{t}}\right)=\frac{t}{2}+S h_{j}\left(v_{A}\right), \text { for all } w_{j} \in \mathcal{W} .
\end{aligned}
$$

Notice that if $A$ is assortative then $\min _{\left(f_{i}, w_{j}\right) \in \mathcal{F} \times \mathcal{W}}\left\{a_{i j}\right\}=a_{11}$. Take now $t_{1}=-a_{11}$. Matrix $A^{t_{1}}$ is assortative and since $S h\left(v_{A}\right) \in C\left(v_{A}\right)$, clearly $S h\left(v_{A^{t_{1}}}\right) \in C\left(v_{A^{t_{1}}}\right)$. Therefore since $a_{11}^{t_{1}}=0$, then $S h_{1}\left(v_{A^{t_{1}}}\right)=0$, for $f_{1} \in \mathcal{F}$. As a consequence of $(20), a_{1 j}^{t_{1}}=0$, for $j=2, \ldots, m$. Similarly, taking $w_{1} \in \mathcal{W}$ we can obtain $a_{i 1}^{t_{1}}=0$, for $i=2, \ldots, m$. Then, we have proved that $a_{i 1}=a_{11}$ for $i=2, \ldots, m$, and $a_{1 j}=a_{11}$ for $j=2, \ldots, m$.

Now players $f_{1} \in \mathcal{F}$ and $w_{1} \in \mathcal{W}$ are null players in $\left(\mathcal{F} \cup \mathcal{W}, v_{A^{t_{1}}}\right)$ since $a_{i j}^{t_{1}}=0$, for $i=1$ or $j=1$, and can be thrown out. Notice that the Shapley value and the core satisfy the strong null property. ${ }^{8}$ Proceed in the same way and the result is achieved.

3. $\longrightarrow$ 1. It is a simple computation taking into account (A.8).

\footnotetext{
${ }^{8} \mathrm{~A}$ solution satisfies the strong null player property if any element of the solution to a game assigns zero to any null player of the game and the solution to a game $\left(N \cup\{i\}, v^{\prime}\right)$ that arises from $(N, v)$ by adding the null player $i$ arises from the solution to $(N, v)$ by adding a zero coordinate for the null player $i$ to any element of the solution to $(N, v)$.
} 


\section{Proof of Theorem 6.1}

Proof. We show that matrix $\widetilde{A}$ given by (22) is assortative and $C\left(v_{A}\right)=$ $C\left(v_{\widetilde{A}}\right)$. First notice that for any $i, j$ such that $|i-j| \leq 1$ we have $\widetilde{a}_{i j}=a_{i j}$, that is, the central strip is the same in both matrices, $A$ and $\widetilde{A}$. It is easy to see that $\widetilde{a}_{i j}+\widetilde{a}_{i+1 j+1}=\widetilde{a}_{i j+1}+\widetilde{a}_{i+1 j}$ for $|i-j| \geq 1$. From here we conclude that the market $(\mathcal{F}, \mathcal{W}, \widetilde{A})$ is supermodular.

We only have to show that $\widetilde{a}_{i j} \leq \widetilde{a}_{k l}$ for $1 \leq i \leq k \leq m$ and $1 \leq j \leq l \leq$ $m$, or equivalently

$$
\begin{aligned}
& \widetilde{a}_{i j} \leq \widetilde{a}_{i+1 j} \text { for } 1 \leq i \leq m-1 \text { and } 1 \leq j \leq m, \text { and } \\
& \widetilde{a}_{i j} \leq \widetilde{a}_{i j+1} \text { for } 1 \leq i \leq m \text { and } 1 \leq j \leq m-1 .
\end{aligned}
$$

We concentrate on (A.11) and the other case (A.10) is proved similarly. Fix $i \in\{1,2, \ldots, m\}$ and we distinguish three cases: (a) $1 \leq i<j \leq m-1$, (b) $i=j$ or $i=j+1$ and (c) $1 \leq j+1<i \leq m$. Cases (a) and (c) come from (22) and the weak-assortative hypothesis on matrix $A$. The case (b) is immediate.

Since both matrices $A, \widetilde{A}$ are supermodular with the same central strip, the equality of the cores follows, see the description of the core for supermodular matrices $(5)-(7)$.

\section{Proof of Theorem 6.2}

Proof. 1. $\longrightarrow 2$. Since $A$ is assortative-solvable, there exists an assortative matrix $B \in \mathrm{M}_{m}^{+}$such that $C\left(v_{A}\right)=C\left(v_{B}\right)$. From here, since matrix $A$ has an optimal matching in the main diagonal, it is clear that the main diagonal is also an optimal matching for matrix $B$ and $b_{k k}=a_{k k}$ for $k=1,2, \ldots, m$. We can assume, maybe by applying Theorem 6.1, that the entries of matrix 
$B$ are given by (22). Now we claim, for all $\left(f_{i}, w_{j}\right) \in \mathcal{F} \times \mathcal{W}$,

$$
b_{i j}=b_{i i}+b_{j j}-v_{B}(\mathcal{F} \cup \mathcal{W})+v_{B}\left(\mathcal{F} \cup \mathcal{W} \backslash\left\{f_{j}, w_{i}\right\}\right)
$$

Trivially (A.12) holds for $1 \leq i=j \leq m$. We discuss one case and the other case is proved similarly. For $1 \leq i<j \leq m$,

$$
\begin{aligned}
b_{i j} & =\sum_{k=i}^{j-1} b_{k k+1}-\sum_{k=i+1}^{j-1} b_{k k}, \\
& =\sum_{k=i}^{j-1} b_{k k+1}+\sum_{k=1}^{i-1} b_{k k}+\sum_{k=j+1}^{m} b_{k k}+b_{i i}+b_{j j}-\sum_{k=1}^{m} b_{k k} \\
& =b_{i i}+b_{j j}-v_{B}(\mathcal{F} \cup \mathcal{W})+v_{B}\left(\mathcal{F} \cup \mathcal{W} \backslash\left\{f_{j}, w_{i}\right\}\right) .
\end{aligned}
$$

Notice that to compute the last equality, matrix without row $j$ and column $i$ is a square assortative matrix and an optimal matching is placed in its main diagonal.

Expression (A.12) can be written as

$$
h_{i j}^{B}=b_{i i}+b_{j j}-b_{i j}, \text { for all }\left(f_{i}, w_{j}\right) \in \mathcal{F} \times \mathcal{W} .
$$

Moreover, by (2) it is easy to see $H(A)=H(B)$.

Now since $B$ is supermodular it is immediate, see (A.13), that matrix $H(B)$ is submodular. To see monotonicity of matrix $H(B)$ we show

$$
\begin{aligned}
& h_{i j}^{B} \leq h_{i+1 j}^{B} \text { for } 1 \leq i \leq m-1 \text { and } 1 \leq j \leq m, \text { and } \\
& h_{i j}^{B} \leq h_{i j+1}^{B} \text { for } 1 \leq i \leq m \text { and } 1 \leq j \leq m-1 .
\end{aligned}
$$

We prove (A.15) and (A.14) is proved similarly. Fix $i \in\{1,2, \ldots, m\}$ and we distinguish three cases: (a) for $1 \leq i<j \leq m-1$ we have $h_{i j}^{B}=$ $b_{i i}+b_{j j}-b_{i j} \leq b_{i i}+b_{j j+1}-b_{i j+1} \leq b_{i i}+b_{j+1 j+1}-b_{i j+1}=h_{i j+1}^{B}$, where the first inequality comes from supermodularity and the second by monotonicity 
of matrix $B$; (b) for $i=j$ or $i=j+1$ just use the monotonicity of $B$; and (c) for $1 \leq j+1<i \leq m$ we use expression (22) to write $b_{i j}=\sum_{k=j}^{i-1} b_{k+1 k}-$ $\sum_{k=j+1}^{i-1} b_{k k}$ and $b_{i j+1}=\sum_{k=j+1}^{i-1} b_{k+1 k}-\sum_{k=j+2}^{i-1} b_{k k}$. Therefore $h_{i j+1}^{B}-h_{i j}^{B}=$ $\left(b_{i i}+b_{j+1 j+1}-b_{i j+1}\right)-\left(b_{i i}+b_{j j}-b_{i j}\right)=b_{j+1 j}-b_{j j} \geq 0$.

2. $\longrightarrow$ 1. Define matrix $B$ by

$$
b_{i j}=a_{i i}+a_{j j}-h_{i j}^{A} \quad \text { for all }\left(f_{i}, w_{j}\right) \in \mathcal{F} \times \mathcal{W} .
$$

Notice first that for $k=1,2, \ldots, m, h_{k k}^{A}=a_{k k}$ and then $b_{k k}=a_{k k}$. We prove that matrix $B$ is assortative and $C\left(v_{A}\right)=C\left(v_{B}\right)$.

First we prove the equality $C\left(v_{A}\right)=C\left(v_{B}\right)$. Let it be $(x, y) \in C\left(v_{A}\right)$. We have, for all $\left(f_{i}, w_{j}\right) \in \mathcal{F} \times \mathcal{W}$,

$$
\begin{aligned}
x_{i}+y_{j} & =\left(a_{i i}-y_{i}\right)+\left(a_{j j}-x_{j}\right)=a_{i i}+a_{j j}-\left(x_{j}+y_{i}\right) \\
& \geq a_{i i}+a_{j j}-v_{A}(\mathcal{F} \cup \mathcal{W})+v_{A}\left(\mathcal{F} \cup \mathcal{W} \backslash\left\{f_{j}, w_{i}\right\}\right)=b_{i j},
\end{aligned}
$$

where we have used the expression of the core in (1). As a consequence, we have for all matching $\mu^{\prime} \in \mathcal{M}(\mathcal{F}, \mathcal{W})$

$$
\sum_{k=1}^{m} b_{k k}=\sum_{k=1}^{m} a_{k k}=\sum_{k=1}^{m} x_{k}+y_{k}=\sum_{\left(f_{i}, w_{j}\right) \in \mu^{\prime}} x_{i}+y_{j} \geq \sum_{\left(f_{i}, w_{j}\right) \in \mu^{\prime}} b_{i j},
$$

which proves that the main diagonal $\mu=\{(1,1),(2,2), \ldots(m, m)\}$ is also an optimal matching for matrix $B$. Therefore we have proved that $C\left(v_{A}\right) \subseteq$ $C\left(v_{B}\right)$.

To see the reverse inclusion, let it be any $(x, y) \in C\left(v_{B}\right)$. Then for all $\left(f_{i}, w_{j}\right) \in \mathcal{F} \times \mathcal{W}$, we have $x_{i}+y_{j} \geq b_{i j}$ and using (A.16) we obtain

$$
x_{j}+y_{i}=\left(a_{j j}-y_{j}\right)+\left(a_{i i}-x_{i}\right) \leq a_{i i}+a_{j j}-b_{i j}=h_{i j}^{A} .
$$

Now taking into account that $v_{A}(\mathcal{F} \cup \mathcal{W})=v_{B}(\mathcal{F} \cup \mathcal{W})$ and the expression of the core in $(1)$, we obtain $(x, y) \in C\left(v_{A}\right)$ finishing the proof. Notice that from (2) we have the equality $h_{i j}^{A}=h_{i j}^{B}$ for all $\left(f_{i}, w_{j}\right) \in \mathcal{F} \times \mathcal{W}$. 
Now we prove that matrix $B$ is assortative. Since by hypothesis matrix $H(A)$ is submodular it is immediate from (A.16) that matrix $B$ is supermodular. To see the monotonicity of matrix $B$ we show

$$
\begin{aligned}
& b_{i j} \leq b_{i+1 j} \text { for } 1 \leq i \leq m-1 \text { and } 1 \leq j \leq m, \text { and } \\
& b_{i j} \leq b_{i j+1} \text { for } 1 \leq i \leq m \text { and } 1 \leq j \leq m-1 .
\end{aligned}
$$

We prove (A.18) and leave the proof of (A.17) to the reader.

Fix $i \in\{1,2, \ldots, m\}$ and we distinguish three cases: (a) for $1 \leq i<j \leq$ $m-1$; (b) for $1 \leq i=j \leq m-1$; and (c) for $1 \leq j<i \leq m$.

Case (a): $1 \leq i<j \leq m-1$.

Notice that for all $1 \leq i<j \leq m$ we have

$$
\begin{aligned}
h_{i j}^{B} & =v_{B}(\mathcal{F} \cup \mathcal{W})-v_{B}\left(\mathcal{F} \cup \mathcal{W} \backslash\left\{f_{j}, w_{i}\right\}\right) \\
& =\sum_{k=1}^{m} b_{k k}-\left[\sum_{k=1}^{i-1} b_{k k}+\sum_{k=i}^{j-1} b_{k} k_{k+1}+\sum_{k=j+1}^{m} b_{k k}\right]=\sum_{k=i}^{j} b_{k k}-\sum_{k=i}^{j-1} b_{k k+1},
\end{aligned}
$$

where we have used the supermodularity of matrix $B$ and the fact that in the square submarket associated to coalition $\mathcal{F} \cup \mathcal{W} \backslash\left\{f_{j}, w_{i}\right\}$ there is an optimal matching on its main diagonal.

As a consequence, for $1 \leq i<j \leq m-1$ a simple computation using (A.16) yields

$$
\begin{aligned}
b_{i j+1}-b_{i j} & =b_{j+1 j+1}-b_{j j}+h_{i j}^{B}-h_{i j+1}^{B} \\
& =b_{j+1 j+1}-b_{j j}+b_{j j+1}-b_{j+1 j+1} \\
& =b_{j j+1}-b_{j j} \\
& =a_{j j}+a_{j+1 j+1}-h_{j j+1}^{A}-a_{j j}=h_{j+1 j+1}^{A}-h_{j j+1}^{A} \geq 0,
\end{aligned}
$$

by the monotonicity of matrix $H(A)$. 
Case (b) for $1 \leq i=j \leq m-1$ is immediate and case (c) for $1 \leq j<$ $i \leq m$ is proved similarly to case (a). 